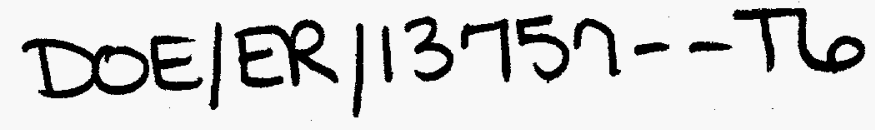

FINAL REPORT

\title{
Fluid Dynamics of Double Diffusive Systems (DE-FG03-87ER13757)
}

\author{
Jeffrey R. Koseff \\ Department of Civil Engineering \\ Stanford University \\ Stanford CA 94305-4020 \\ 415-723-9032 \\ koseff@ce.stanford.edu (internet)
}

\section{OVERALL GOALS}

Over the past seven years we have conducted an experimental, numerical, and theoretical study of the stability of doubly diffusive systems, and of mixing processes in stratified turbulence. For the study of the stability of doubly diffusive systems continuous gradients of two diffusing components (heat and salinity in our case) were used as the initial condition, and forcing was introduced by lateral heating and surface shear. The goals of this work included (1) quantification of the effects of finite amplitude disturbances on stable, double diffusive systems, particularly with respect to lateral heating, (2) development of an improved understanding of the physical phenomena present in sheardriven flows in doubly diffusive stratified environments, (3) increasing our knowledgebase on turbulent flow in stratified environments and how to represent it, and (4) formulation of a numerical code for such flows.

In particular, the overall goals of this aspect of the research were as follows:

1) develop more general stability and scaling criteria for the destabilization of doubly-stratified systems,

2) study the variation of flow structure and scales with Rayleigh ratio and lateral heating ratio,

3) delineate the mechanisms governing convective layer formation and merging,

4) study the mixing processes within the convective layers and across interfaces, and estimate the heat and mass fluxes in such a system,

5) quantify the effects of turbulence and coherent structures (due to a winddriven surface shear) on a doubly stratified system, and

6) study the interaction between surface shear and side-wall heating destabilization mechanisms.

We successfully completed goals 1 through 4, the results of which are described below (Results) and in the papers listed under Publications. In the course of beginning our research to address goals 5 and 6 it became apparent that other fundamental questions 


\section{DISCLAIMER}

This report was prepared as an account of work sponsored by an agency of the United States Government. Neither the United States Government nor any agency thereof, nor any of their employees, makes any warranty, express or implied, or assumes any legal liability or responsibility for the accuracy, completeness, or usefulness of any information, apparatus, product, or process disclosed, or represents that its use would not infringe privately owned rights. Reference herein to any specific commercial product, process, or service by trade name, trademark, manufacturer, or otherwise does not necessarily constitute or imply its endorsement, recommendation, or favoring by the United States Government or any agency thereof. The views and opinions of authors expressed herein do not necessarily state or reflect those of the United States Government or any agency thereof. 


\section{DISCLAIMER}

Portions of this document may be illegible in electronic image products. Images are produced from the best available original document. 
needed to be answered first. As a result we began a related, but different, study over the final two years of the project.

The basis for this study was our desire to gain a more complete understanding of the role of molecular diffusion (and in particular its magnitude relative to viscosity, as represented by the Schmidt Number) on the efficiency of mixing in stratified, turbulent flows. Since molecular diffusion controls the irreversible mixing of heat and mass and stratification can inhibit the ability of turbulence to stir a fluid, both the molecular diffusivity and stratification affect the net transport of mass and heat in many turbulent, stratified flows. A more complete understanding of how turbulent stirring, molecular diffusion, and gravitational adjustment interact is essential to predicting the mixing of . discharges from power plants, the efficiency of solar ponds, and the storage and transport of heat and greenhouse gases in the ocean's surface waters.

Problems with direct measurement of the turbulent fluxes needed to compute net mass transport lead most researchers to use indirect methods that rely on concepts such as mixing efficiency and on knowledge of the small-scale structure of the turbulence. However, the effects of stratification and the molecular diffusivity on mixing efficiency remain unclear, and a full evaluation of the small-scale turbulent structure in a stratified environment is lacking. In addition, incomplete knowledge of the evolution of the turbulence introduces uncertainty into the sampling of the fluxes and the calculation of the net mass transport. To address these problems, we attempted to:

1. Determine the effect of stratification on the mixing efficiency by reconciling previous predictions and by studying the behavior for strong stratifications;

2. Characterize the effect of the molecular diffusivity on the mixing efficiency;

3. Examine the small-scale structure of stratified turbulence and evaluate assumptions made by field researchers pertaining to measurements of dissipation;

The basis for much of the work was a scaling analysis we developed to predict the mixing efficiency behavior over a range of grid Richardson $\left(\mathrm{Ri}_{0}\right)$ numbers. The analysis comprises computing the potential energy change from the mean density equation and estimating an integral of the vertical mass flux with scaling for the vertical overturn scale and the decay time of the turbulence. We performed towed grid turbulence experiments in the laboratory that allow us to check our scaling for part of the $\mathrm{Ri}_{0}$ range. However, since the strong stratifications needed to check the scaling for higher Richardson numbers are difficult to achieve with experiments, we also used linear theory analysis to check the predictions in this regime.

\section{RESULTS}

\section{Stability of Doubly Diffusive Systems}

We have analyzed the results from a group of experiments in which a uniform, lateral heat flux was applied to opposing linear gradients of temperature and salinity. The Rayleigh ratio, $R_{p}$, and the lateral heating ratio, $R l$, are used to provide a measure of the relative stability of the experimental system for a given heating rate and set of stratification conditions. The intrusions that form at the heated endwall have been grouped into three classes of flow depending on their characteristic thickness, propagation velocity, and vertical structure as defined by profiles of temperature and salinity. Intrusions in Class I 
were found to be smaller and less dynamic than those in Classes II and III and appear to be similar in structure to intrusions observed when heating is applied to a single component stratification.

Plots of temperature vs. salinity (T-S) were used to identify regions of diffusive, finger, and gravitational instabilities from the vertical profiles. Based on the T-S diagrams, fingering is most prominent in Class II flows, a result which is confirmed by flow visualization. However, the diagrams indicate that fingers can also develop in Class I flows, although they have not been observed visually in this class. In both Classes I and II, the fingers contribute to more rapid mixing of salt than heat within the intrusions. In Class III, however, both temperature and salt mix rapidly (relative to Classes I and II) indicating that convective overturning is the primary mechanism of vertical mixing in this class.

A theoretical expression was developed for the initial thickness, $h$, of the intrusions. The analysis is valid for $R l » 1$, but experiments indicate that it gives a reasonable estimate of $h$ for cases when $R l>5$. All attempts to develop an analogous expression for $R l \sim 1$ were unsuccessful due to the increased complexity of the analysis in this parameter range.

Merging processes were found to be dominated by horizontal motions in Class I. Weaker intrusions tend to get forced back towards the heated endwall by strong return flows and eventually become entrained by their neighbors above and below. In contrast, merging in Classes II and III is dominated by strong vertical motions at the heated endwall. The higher relative heating rates in these classes generate active vertical mixing close to the endwall which serves to combine neighboring cells. In all classes, the final thickness of the layers ranges from 2-4 times the initial intrusion thickness. Richardson number fields indicate that interfacial shear is not likely to contribute to mixing across the density interfaces away from the endwall in any of the observed flows.

Calculations of Thorpe scales within the intrusions indicates that the scale of the largest overturns is about 10-30\% of the layer thickness in Classes II and III and 10-25\% in Class I. These estimates agree very well with observations from flow visualization. Application of the long box analysis (presented by Jeevaraj and Imberger, Journal of Fluid Mechanics 1991) to our experiments did not produce reasonable agreement. This is because our flows were characterized by significant vertical motions (in the form of fingers and convective overturns) which contributed to much more vertical mixing than predicted by the long box analysis. However, reasonable agreement could be obtained by using values of the diffusivities which were several times larger than the molecular values. The discrepancies between our experimental results and those of Jeevaraj and Imberger are attributed to differences in the method of heating between the two studies.

For Class III intrusions, a blocking mechanism is described whereby selfpropagation can occur when the initial value of $R_{\rho}$ is close to critical stability. Although experimental evidence supports the existence of a blocking mechanism, it is likely that additional factors such as surface cooling and nonlinear gradients contributed to the continued propagation of the intrusions in the two observed Class III experiments.

\section{Mixing Processes in Stratified Turbulent Flows}

The main results of this work involve predictions of the effect of stratification on the mixing efficiency with both scaling and linear theory, an assessment of the mixing efficiency differences for heat-stratified and salt-stratified water, and a proposal for evaluating the effects of the Richardson, Reynolds, and Schmidt numbers on the mixing:

A simple theory based on a direct calculation of the mean potential energy change and the multiple-regime model of the turbulence behavior predicts the effect of stratification 
on the mixing efficiency. For grid turbulence in a linearly-stratified fluid the theory has reasonable success. For weak to moderate stratifications, the theory reproduces and unifies predictions from previous researchers and suggests when each prediction should hold. For the less well-studied case of strong stratification, the theory predicts that the efficiency should decrease.

Experiments and linear theory support some of the predictions of the scaling. The experiments reproduce the regime II behavior fairly well, and regime III seems to begin at the value of $\mathrm{Ri}_{0}$ suggested by the scaling. A linear theory similar to rapid distortion theory provides predictions for the strongly-stratified cases not accessible with experiments. An application of the theory that accounts for the finite extent of the fluid predicts a decrease in the efficiency as the grid Richardson number becomes large, consistent with the scaling. Although the linear theory should not apply for weak stratifications, it reproduces the regime I and regime II behavior surprisingly well.

Within the error and uncertainty of the experiments the efficiencies in heat-stratified water and salt-stratified water match for moderate stratifications $\left(10^{-2}<\mathrm{Ri}_{0}<3 \times 10^{-1}\right)$. In principle, a complete study of the Schmidt number effects would use a wide range of stratifiying agents; however, the $\mathrm{Sc}=7$ and $\mathrm{Sc}=700$ cases are the most important cases for oceanographic applications.

A time scale comparison offers an explanation for the agreement of the efficiencies, and it allows several other observations to be made. Its predictions are consistent with several experimental observations, and it suggests that analyses of mixing in fluids with Sc $>1$ should consider Reynolds and Schmidt number effects separately. The time scale comparison also provides numerical estimates of the time scales involved in mixing. These estimates can be used to refine or understand similar theories in more detail.

\section{PUBLICATIONS}

The following publications acknowledge the support provided by the grant DE-FG0387ER13757.

\section{Ph.D Dissertations}

Rehmann, Chris R., " Effects of Stratification and Molecular Diffusivity on the Mixing Efficiency of Decaying Grid Turbulence", Ph.D. dissertation, Department of Civil Engineering, Stanford University, Stanford, CA, 1995.

Thomas McDonald, E., " The Dynamics and Structure of Lateral Intrusions in a Continuously Stratified Heat/Salt System", Ph.D. dissertation, Department of Civil Engineering, Stanford University, Stanford, CA, 1992, 287p.

\section{Refereed Journals}

Thomas McDonald, E., and Koseff, J.R., " The Internal Structure of Lateral Intrusions in a Continuously Stratified Heat/Salt System", Physics of Fluids, 6 (12), 1994, pp.3870-3883.

Schladow, S.G., Thomas, E., and Koseff, J.R., "The Dynamics of Lateral Intrusions in a Thermohaline Stratification," Journal of Fluid Mechanics, 237, 1992, pp. 127-165. 
Schladow, S.G., "Oscillatory motion in a side-heated cavity ", Journal of Fluid Mechanics, 213, 1990, pp. 589-610.

Schladow, S.G., Patterson, J.C. and Street, R.L. "Transient flow in a side-heated cavity: a numerical study", Journal of Fluid Mechanics, 200, 1989, pp. 121-148.

\section{Conference Proceedings}

Rehmann, C.R. and Koseff, J.R., "Mixing efficiency of decaying grid turbulence in a stratified fluid." 4th Int. Symp. Strat. Flows, Grenoble, France, June, 1994.

Thomas McDonald, E., and Koseff, J.R., " Experimental Observations of 'Self-Propagating' Intrusions in a Laterally Heated Double Diffusive System", Proc. of AGU Chapman Conference on Double Diffusion, Nov 3-6. 1993.

Thomas McDonald, E., and Koseff, J.R., "The Structure of Lateral Intrusions in a Two Component Continously Stratified Heat/Salt System," presented at the AGU Ocean Sciences Meeting; Paper No. 011B-11, New Orleans, LA., 1992.

Thomas McDonald, E., and Koseff, J.R., "On Self-Propagation of Thermohaline Intrusions," Proc. Tenth Symposium on Energy Engineering Sciences, Argonne National Laboratory, Argonne, IL, May 11-13, 1992, pp. 60-67.

Thomas, E., Schladow, S.G., and Koseff, J.R., "Thermohaline Intrusions in a Two Component Density Stratified System," Proc. of Int'l Conf. on Physical Modeling of Transport and Dispersion, Mass. Inst. Tech., Aug 7-10, 1990, pp. 8C.7-12.

Schladow, S.G., Thomas, E., and Koseff, J.R., "Structure of Thermohaline Intrusions--A Comparison of Numerical and Experimental Results," EOS, 71, No. 43, p. 1360, 1990.

Thomas, E., Schladow, S.G., and Koseff, J.R., "The Formation of Lateral Intrusions in a Thermohaline Stratification," Proc. Seventh Symposium on Energy Engineering Sciences, Argonne National Laboratory, June 19-21, 1989, pp. 137-144.

Schladow, S.G. and Street, R. L."Transient flow in a weakly stratified side-heated cavity", ASME FED 71, 1988, pp. 39-41.

Thomas, E., Schladow, S.G., and Koseff, J.R., '"Thermohaline Intrusions in a Two-Component, Density Stratified System," AGU Fall Meeting, Paper No. 051A-11, San Francisco, CA, 1989, pp. 1167. 